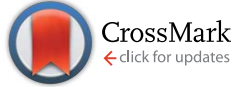

Cite this: RSC Adv., 2015, 5, 63745

Received 19th May 2015

Accepted 21st July 2015

DOI: $10.1039 / c 5 r a 09406 b$

www.rsc.org/advances

\section{In vivo reversal of general anesthesia by cucurbit[7]- uril with zebrafish models $\dagger$}

\author{
Huanxian Chen,,$^{\mathrm{a}}$ Judy Y. W. Chan, $\dot{t}^{\mathrm{a}}$ Shengke Li, ${ }^{\mathrm{a}}$ Jessica J. Liu, ${ }^{\mathrm{b}}$ Ian W. Wyman, ${ }^{\mathrm{b}}$ \\ Simon M. Y. Lee, ${ }^{a}$ Donal H. Macartney ${ }^{b}$ and Ruibing Wang ${ }^{* a}$
}

A general anesthetic is a drug that brings about a reversible loss of consciousness, during a surgical or therapeutic procedure to render the patient free of pain and anxiety. However, the effect of anesthetics may linger far beyond the necessary time required and induce adverse effects. In addition, many surgical patients need to recover to a conscious state that allows them to make important decisions soon after their surgery. Unfortunately, there are currently no clinically-available anti-dotes to reverse the effects of anesthetics. In this study, we demonstrate the in vitro supramolecular host-guest complexations between macrocyclic cucurbit[7]uril (CB[7]) and a commonly used general anesthetic in fish, tricaine mesylate (TM), and we report for the first time the in vivo reversal effect of $\mathrm{CB}$ [7] to general anesthesia induced by TM with zebrafish models. These findings might lead to a new approach that may allow patients to regain lucidity much faster than their natural recovery from general anesthesia, and may also be used to reverse potentially life-threatening toxic effects encountered by some patients in response to general anesthesia.

\section{Introduction}

General anesthesia is a medically induced reversible coma resulting from the administration of one or more general anaesthetic agents. ${ }^{1}$ Anesthetics are often used during a surgical or therapeutic procedure to provide relief of pain and anxiety. ${ }^{2}$ The recovery from general anesthesia has been a passive process whereby the patients recover from the anesthesia on their own and currently no reversal agents are available to actively induce recovery. ${ }^{3}$ Very often, the effects of anesthetics linger far beyond the time required and some of these effects even remain after patients awaken from surgery. For example, they are usually groggy and disoriented, and it can take hours for a patient to fully recover their cognitive skills. In reality, many surgical patients need to recover to a clearheaded state that allows them to make important decisions regarding their medical treatment soon after their surgery. ${ }^{3}$ In addition, some patients unexpectedly experience marked sensitivity to these drugs that can include acute fatal toxicity, long-term

\footnotetext{
${ }^{a}$ State Key Laboratory of Quality Research in Chinese Medicine, Institute of Chinese Medical Sciences, University of Macau, Taipa, Macau SAR, China. E-mail: rwang@ umac.mo; Tel: +853-8822-4689

${ }^{b}$ Department of Chemistry, Queen's University, Kingston, ON, K7L 3N6, Canada $\dagger$ Electronic supplementary information (ESI) available: ESI-MS spectrum of TM-CB[7] complex, representative figures showing larval zebrafish swimming patterns under different treatment conditions, fluorescent image of zebrafish heart, cardiac functions of zebrafish larvae at 20 minutes after recovery, fish anesthesia and recovery stages. See DOI: 10.1039/c5ra09406b

$\$$ These authors contributed equally to this work.
}

neurotoxicity, and cardiotoxicity, requiring further medical availability of drugs to reverse the effects of the anesthetic when anesthesia is no longer needed. Unfortunately, there are currently no effective anti-dotes to reverse the effects of anesthetic agents in clinics although the use of stimulant drugs have been attempted with animal models to awaken certain areas of the brain to help induce emergence from anesthesia. ${ }^{3,5}$ For instance, methylphenidate has been demonstrated with in vivo rat models to actively promote emergence from isofluraneinduced general anesthesia by increasing arousal and respiratory drive, possibly through activation of dopaminergic and adrenergic arousal circuits. ${ }^{3}$ However, this approach wouldn't remove the residual anesthetic agents within the body that would continue to work on inducing unconsciousness. Consequently, this drug does not truly "reverse" the anesthetic agent; instead it simply awakens certain areas of the brain while the other regions remain anesthetized. In this case, the inherent adverse effects of general anesthesia, such as neurotoxicity and cardiotoxicity, would still be present as the remaining anesthetic agent is not removed. Moreover, there are additional toxicities and potential addiction issues associated with the use of arousal agents. ${ }^{6}$ As a result there is a real need to develop alternative classes of reversal agents that can actively function to bring about a timely recovery from general anesthesia by deactivating or removing anesthetic agents from the blood plasma.

Interestingly, in the field of anesthesia, a novel approach has been proposed for over a decade to reverse the activity of assistance. ${ }^{4}$ An ideal solution to these situations would be the 
steroidal neuromuscular blocking agents (NMBAs) by providing a host molecule that will selectively bind to free NMBAs in the plasma. $^{7}$ The resultant concentration gradient would cause a movement of the neuromuscular junction (NMJ)-bound NMBA into the plasma where it can be encapsulated by more of the host molecule, rapidly terminating the neuromuscular blockade. $^{8}$ One of the successful examples of this class of reversal agents to NMBA is Sugammadex, which is a derivative of $\gamma$-cyclodextrin that can selectively complex with several NMBAs with fairly large binding constants (on the order of $10^{6}$ to $\left.10^{7} \mathrm{M}^{-1}\right) .{ }^{8}$ In addition, acyclic analogs of cucurbit $[n]$ uril $(\mathrm{CB}[n], n=5-8,10$, and 14$)$ type molecular containers have demonstrated similar reversal effects on steroidal NMBAs in vivo. ${ }^{9,10} \mathrm{CB}[n] \mathrm{s}$ are macrocyclic compounds consisting of $n$ glycoluril units that are bridged by $2 n$ methylene groups, and they possess a hydrophobic internal cavity that is located between two carbonyl lined portals. In comparison with cyclodextrins, $\mathrm{CB}[n] \mathrm{s}$ can encapsulate cationic and neutral molecules with much higher selectivity and binding affinities. ${ }^{11}$ Among all of the members of the $\mathrm{CB}[n]$ series, $\mathrm{CB}[7]$ (Fig. 1, left) in particular has drawn considerable interest because of both its good watersolubility and its capacity to encapsulate organic molecules of a wide range of sizes. It has been demonstrated by numerous research groups including ours that $\mathrm{CB}[7]$ can effectively encapsulate a variety of drugs and bioactive molecules, including atenolol (a beta-blocker), ${ }^{12}$ pyrazinamide (a tuberculosis drug), ${ }^{13}$ platinum-based anti-cancer drugs such as cisplatin and others, ${ }^{\mathbf{1 4 - 1 6}}$ prilocaine and various other local anaesthetic, ${ }^{17}$ as well as ranitidine and coumarin. ${ }^{18,19}$ In addition, we have also actively investigated the complexation behaviors of steroidal NMBAs ${ }^{20}$ and local anesthetic agents ${ }^{17}$ by $\mathrm{CB}[7]$ and reviewed the use of $\mathrm{CB}[n]$-type hosts for the reversal of steroidal NMBAs. ${ }^{8}$ However, macrocyclic $\mathrm{CB}[n]$ hosts have never been reported for their complexations with general anesthetic agents and there are no precedent examples demonstrating their reversal activities to general anesthesia. With this knowledge and experience in hand, and in consideration of the fact that there have been no effective reversal agents available to counteract general anesthesia, we decided to evaluate the general anesthesia reversal capabilities of $\mathrm{CB}[7]$ using in vivo zebrafish models.

Zebrafish (Danio rerio) have become a powerful and costeffective model for drug screening and efficacy evaluation. ${ }^{21}$ Several advantages provided by zebrafish models, including their rapid and synchronous growth as well as the transparency of their chorion and bodies that enable non-invasive and direct

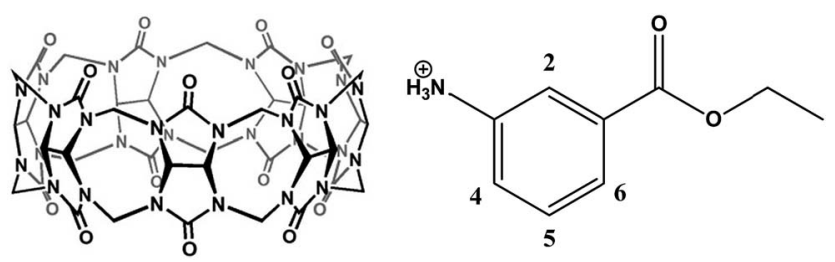

Fig. 1 The molecular structures of $\mathrm{CB}[7]$ (left) and tricaine (right, mesylate counterion is not shown). observations of their organs, have been well-recognized. ${ }^{22}$ Previous reports have shown that the biological responses of zebrafish to small molecules and drugs are similar to those exhibited by mammals. ${ }^{23,24}$ These features suggest that zebrafish shall provide an useful model for the evaluation of the reversal effect of $\mathrm{CB}[7]$ to general anesthesia that may parallel that of humans. Functional and behavioral studies of zebrafish focusing on various parameters such as hearing, learning and locomotion have also been developed that are useful for identifying neurotoxins without obvious phenotypic changes. ${ }^{25,26}$ In addition, organ-specific toxicity models based on zebrafish have been well established by a few research groups including ours. ${ }^{22,27}$

Tricaine mesylate (TM, also known as MS-222, Fig. 1, right) is the most commonly used general anesthetic in fish and the only one that has been approved by the US Food and Drug Administration. TM has been used extensively for immobilizing fish, amphibians, and other aquatic cold-blooded animals with well-understood efficacy and safety profiles. ${ }^{28}$ Previous studies focusing on the mechanism by which TM exerts its anesthetic action have suggested that TM blocks sodium ion and to a lesser degree potassium ion channels in nerve membranes, thus limiting nerve membrane excitability. ${ }^{7}$ Action potentials and spontaneous contractions of muscles are eliminated by TM, including sensory input and reflexes, ${ }^{28-31}$ although one of the most recent studies has suggested that TM only efficiently blocks neural action potential but does not prevent directly evoked muscle contraction. ${ }^{32}$ As is the case with humans, a prolonged recovery from an anesthetic can potentially cause fish to experience weakened respiration, hypoxia, and finally, respiratory and cardiac collapse. ${ }^{33}$

Herein we report for the first time the in vitro host-guest complexation of $\mathrm{CB}[7]$ and $\mathrm{TM}$, and the in vivo reversal effects of $\mathrm{CB}[7]$ to $\mathrm{TM}$-induced general anesthesia among zebrafish models. This discovery suggests that the molecular container $\mathrm{CB}$ [7] may be clinically useful as an agent to reverse general anesthetic-induced unconsciousness and its associated adverse effects. These findings will not only open up a new window for the design of novel anesthesia reversal agents, but will also further expand the use of $\mathrm{CB}[n]$ hosts in the biomedical field.

\section{Results and discussion}

\subsection{The host-guest complexation between $\mathrm{CB}[7]$ and TM}

Initially, we investigated the supramolecular host-guest complexation between $\mathrm{CB}[7]$ and $\mathrm{TM}$ in order to understand important binding parameters such as binding geometry, stoichiometry and affinity. Upon the addition of $\mathrm{CB}$ [7] to a deuterated aqueous solution of TM, all of the guest proton resonances of tricaine cation were observed to exhibit upfield shifts in the ${ }^{1} \mathrm{H}$ NMR spectrum (with limiting chemical shifts varying from 0.32 to $0.84 \mathrm{ppm}$ ), indicative of the encapsulation of the whole tricaine cation inside the cavity of $\mathrm{CB}$ [7] (Fig. 2). Conversely, the lack of a change in the chemical shift of the resonance for the methyl protons of the mesylate counteranion implies that the mesylate did not undergo complexation with $\mathrm{CB}$ [7], presumably 
due to repulsion between its negative charge and the carbonyl portals of the host. ${ }^{19}$

In addition to ${ }^{1} \mathrm{H}$ NMR spectral characterization, we also conducted molecular modeling (MM2 energy-minimization) to obtain further evidence of the existence of the $1: 1$ host-guest complexes, and verify the formation of inclusion complexes as well as the guest binding geometry within the host cavity. The modelled structure of the $\mathrm{CB}[7]$ host-guest complex with TM is shown in Fig. 3a. The entire aromatic ring of the guest is preferentially encapsulated within the $\mathrm{CB}[7]$ cavity placing the positively charged ammonium nitrogen atom adjacent to the portal, in line with the carbonyl groups for optimal cationdipole interactions. This orientation of the host-guest complex places the ethyl group outside of the portal, although the ethyl proton resonances exhibit complexation-induced upfield shift changes in the ${ }^{1} \mathrm{H}$ NMR spectra (Fig. 1). The contradictory observation is likely a result of molecular shuttling of the guest within the cavity of the host, such that the full length of the tricaine is inside the cavity, on average. Either limit of the complexation geometry places the ester carbonyl group within the cavity and aligning its dipole with the quadrupolar moment of the $\mathrm{CB}[7]$. We have previously observed evidence for this weak orienting interaction with $\mathrm{CB}[7]$ complexes of a number of ketone guest molecules. ${ }^{34}$

The $1: 1$ binding stoichiometry was also verified by our Job's plot that was derived from a continuous variation titration method. ${ }^{35}$ The Job's plot for the TM-CB[7] system (with [CB[7]] + [TM] fixed at $1 \mathrm{mM}$ ), as monitored via UV-visible spectroscopy (Fig. $3 \mathrm{~b}$ ) at $270 \mathrm{~nm}$ reached a maximum at a ratio of 0.50 for [CB $[7]] /[\mathrm{CB}[7]]+[\mathrm{TM}]$. This behavior thus confirmed that the $1: 1$ complex between $\mathrm{CB}[7]$ and $\mathrm{TM}$ represented the dominant species in this concentration range. The 1:1 host-guest complexation was further confirmed by high resolution ESI-MS analysis, which revealed a singly charged species at $\mathrm{m} / \mathrm{z}$ of 1328.3503 (ESI Fig. S1 $\dagger$ ), while the calculated $\mathrm{m} / \mathrm{z}$ value for TM-CB[7] is 1328.4303 .

In addition to the evidence provided by the Job's plot, the formation of $1: 1$ inclusion complexes between $\mathrm{TM}$ and $\mathrm{CB}[7]$ in aqueous solution was also suggested by UV-visible absorbance measurements of TM $(1 \mathrm{mM})$ as it was titrated with increasing amounts of $\mathrm{CB}[7]$. The gradual addition of $\mathrm{CB}[7]$ to a buffered aqueous solution of TM resulted in decreases in the peak at $308 \mathrm{~nm}$ (Fig. 4) in line with the inclusion of the guest within the macrocyclic microenvironment that differed from the bulk surroundings. The non-linear least-squares fit (Fig. 4, inset) is consistent with a $1: 1$ binding stoichiometry model and provides a binding constant $K_{\mathrm{a}}$ of $(8.0 \pm 0.5) \times 10^{4} \mathrm{M}^{-1}$. This binding affinity is moderate, and is comparable in strength with several other cationic species complexed by $\mathrm{CB}[7] .{ }^{36}$ In clinical settings, infusion/inhalation of anesthetics is usually stopped immediately after surgery, and thus the remaining anesthetic concentration is relatively low. The addition of a relatively high concentration of $\mathrm{CB}[7]$ (due to its high biocompatibility and low toxicity $^{22}$ should be able to compensate for the relatively moderate binding affinity.

With a fundamental understanding of the host-guest complexation behavior between $\mathrm{CB}[7]$ and $\mathrm{TM}$ in hand, we moved forward to test the ability of $\mathrm{CB}[7]$ to reverse the general anesthesia in vivo.

\subsection{Locomotion recovery from anesthesia}

It has been very well known that anesthetic agents affect locomotion behaviors of zebrafish even during their recovery from

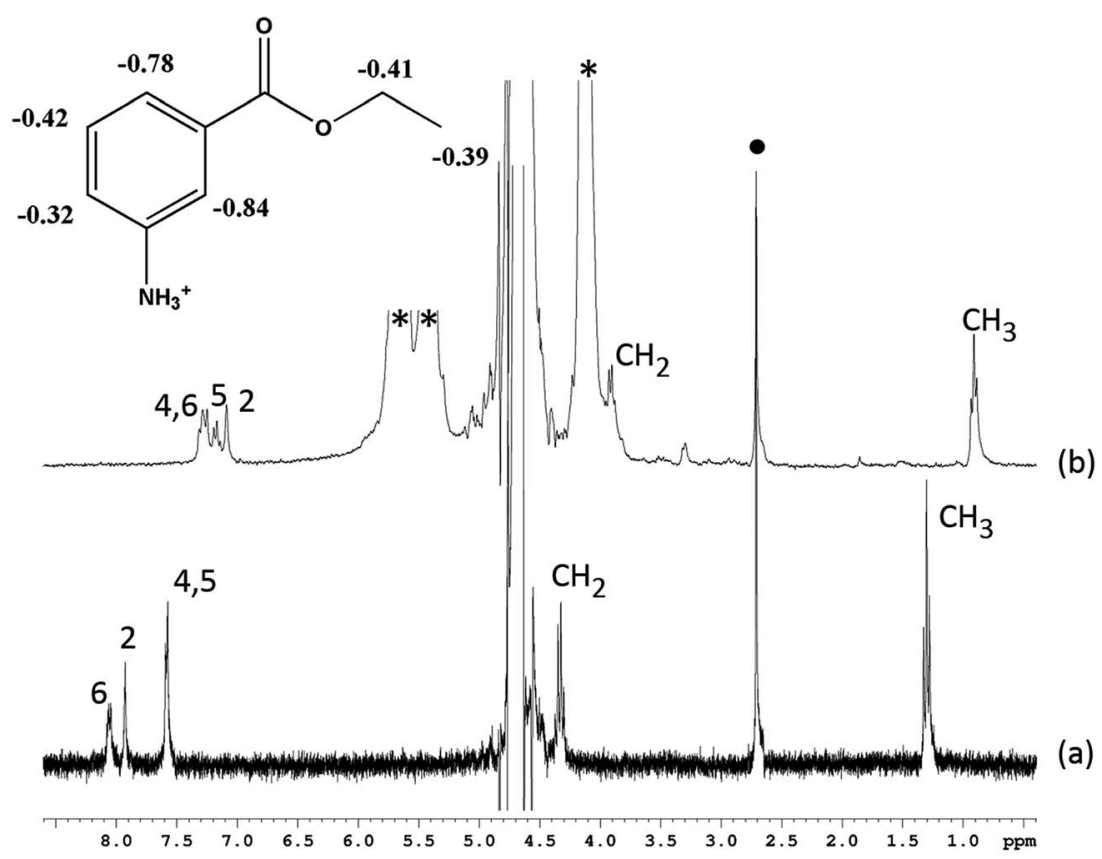

Fig. $2{ }^{1} \mathrm{H}$ NMR spectra of TM in the absence (bottom) and in the presence of 5 equiv. (top) of CB[7]. * indicates the protons of CB[7] and indicates mesylate protons. The insert tricaine chemical structure was labeled with limiting chemical shifts for each of the proton. 


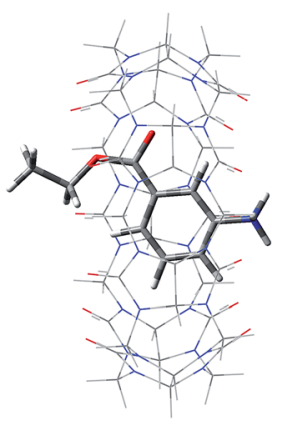

(a)

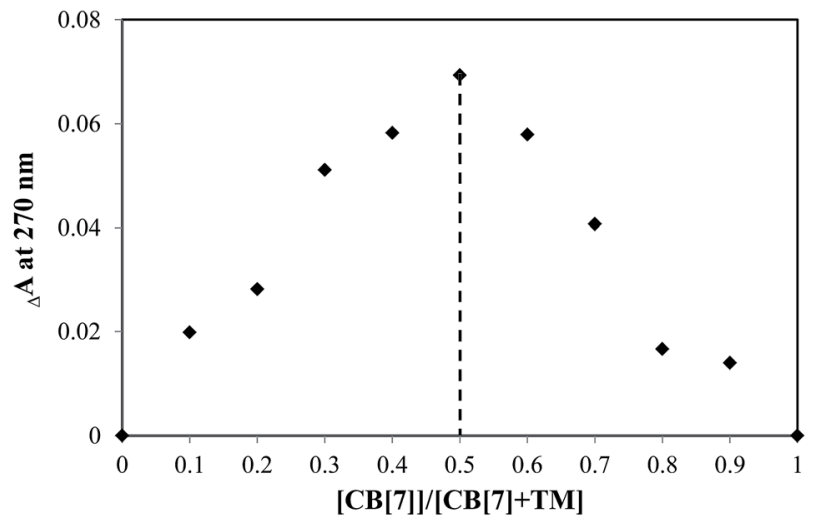

(b)

Fig. 3 Side-view of the MM2 energy-minimized model of the TM-CB[7] complex (a), and the Job's plot of the CB[7]-TM host-guest complexes prepared via the continuous variation titration method and monitored by the changes in the UV-visible absorbance at $270 \mathrm{~nm}$ (b).

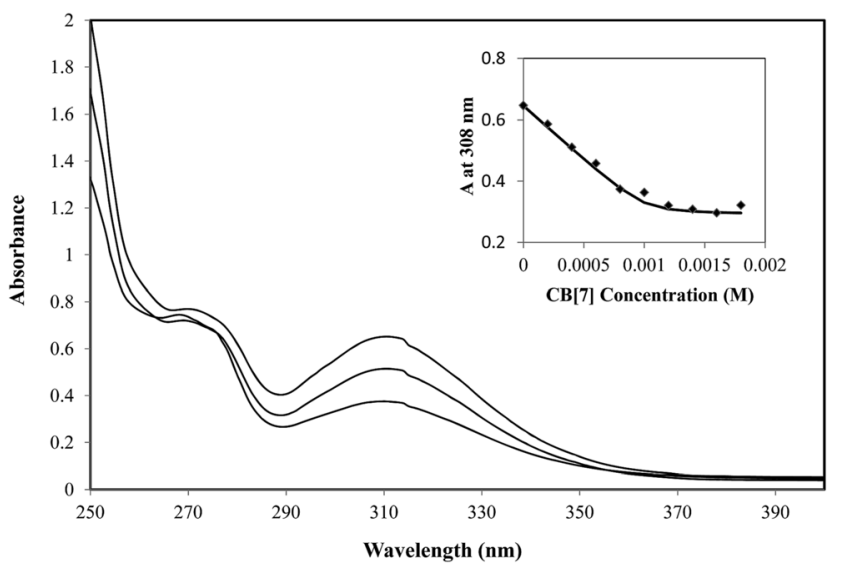

Fig. 4 UV-visible spectra of TM $(1 \mathrm{mM})$ in the presence of increasing amounts of CB[7] (a: 0, b: 0.6, c: 1.2 equiv.) in E3 buffered aqueous solution. Insert: dependence of the absorbance at $308 \mathrm{~nm}$ as a function of the CB[7] concentration.

anesthesia, including their swimming velocity and distance. ${ }^{37}$ In this study, zebrafish larvae were employed to investigate the ability of $\mathrm{CB}[7]$ to reverse the anesthesia induced by TM. A previous study has shown that $\sim 1 \mathrm{mM}$ of TM induced stage 4 anesthesia or beyond within $3 \mathrm{~min}$ (ESI Tables 1 and $2 \uparrow$ for staging system of anesthesia and recovery). ${ }^{38}$ With this in mind, we anesthetized the zebrafish for 3 min with $1 \mathrm{mM}$ TM in E3 medium. As expected, subjecting the zebrafish to $3 \mathrm{~min}$ of treatment with $1 \mathrm{mM}$ TM induced a stage 4 anesthesia, which was characterized by loss of balance, loss of response to spinal reflex, and by much slower opercular movement. Right after the zebrafish were anesthetized, TM was removed and added was $\mathrm{E} 3$ medium or $0.5 \mathrm{mM} \mathrm{CB}[7]$ in $\mathrm{E} 3$ medium, and the locomotion behaviors were immediately monitored. Fig. 5 shows the total swimming distances that were observed during each $10 \mathrm{~min}$ interval as the zebrafish recovered from anesthesia. During the first $10 \mathrm{~min}$, the addition of $\mathrm{CB}[7]$ resulted in a nearly complete recovery of locomotion behavior, comparable with the control groups (non-anesthetized groups), in significant contrast to the natural recovery group. Similar results were observed during the second $10 \mathrm{~min}$ interval, indicating that the zebrafish in the natural-recovery group were still under recovery. Until the fifth $10 \mathrm{~min}$ interval, the anesthetized natural-recovery group swam comparable distances with the other groups, including the nonanesthetized control groups (for representative swimming patterns in each group, see ESI Fig. S2 $\dagger$ ). These findings suggest that $\mathrm{CB}[7]$ accelerated the locomotion recovery of anesthetized fish to only $10 \mathrm{~min}$ or less, whereas the natural recovery group required at least $40 \mathrm{~min}$ to reach a comparable recovery. In addition, it should be noted that the addition of $0.5 \mathrm{mM} \mathrm{CB}[7]$ to the non-anesthetized fish had little influence on their locomotion behaviors when they were compared to the nonanesthetized control group.

\subsection{Cardiac functions recovery from anesthesia}

A growing number of non-cardiac drugs, particularly anesthetic agents, have been shown to block sodium channels and thus carry an increased risk of adverse cardiovascular events in patients..$^{39}$ Several reports suggested that voltage gated sodium channel blockers are able to decrease heart rate and cardiac output in anesthetized animals, ${ }^{40,41}$ which would in turn increase the risk of accidental death during anesthesia. ${ }^{29}$ Previous studies have described a reduction in stroke volume and cardiac output measured indirectly using aortic blood flow, and reported significant depression of cardiac contractility during TMinduced fish anesthesia in zebrafish larvae. ${ }^{42,43}$ Therefore, we evaluated the cardiac function of zebrafish larvae post TMinduced anesthesia, in the absence and presence of $\mathrm{CB}[7]$.

Consistent with the previous reports, ${ }^{30,31,42,43}$ our study showed that the heart rate (HR), stroke volume (SV), cardiac output (CO), and fractional shortening (FS) had all decreased to different degrees after anesthesia of $4 \mathrm{dpf}$ zebrafish with $1 \mathrm{mM}$ TM for 3 min. After the TM had been replaced with E3 medium or $\mathrm{CB}[7]$ in $\mathrm{E} 3$ medium, the zebrafish began to recover from anesthesia. Through exploration of the time point in our pilot experiments, we chose to monitor cardiac function of zebrafish 


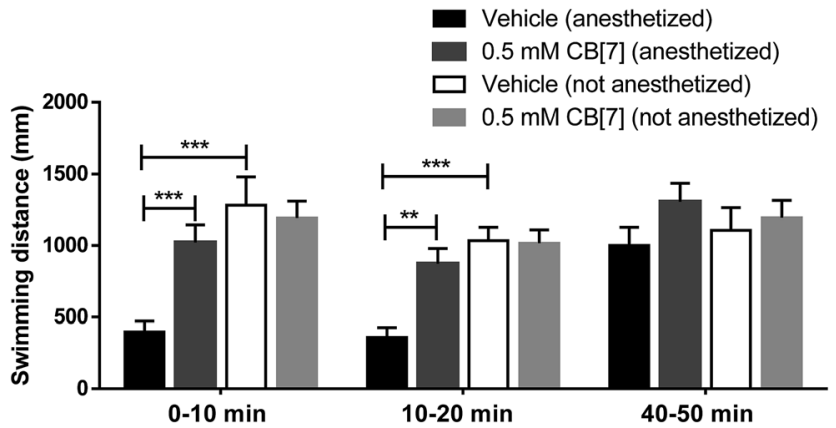

Fig. 5 The total swimming distance of each zebrafish observed during each 10 min time interval as they recovered from anesthesia. These measurements were recorded in the presence and in the absence of $0.5 \mathrm{mM} \mathrm{CB}$ [7] in E3 medium. Non-anesthetized zebrafish treated with or without CB[7] were used as the control groups. Data are expressed as mean \pm S.E.M. ( $n=8$ in each group). ${ }^{* * P} P<0.01, * * * P<0.001$.

at fifth min post anesthetic treatment, when the anesthetized zebrafish (in both natural-recovery group and $\mathrm{CB}[7]$ treated groups) were still in the recovery process. As shown in Fig. 6, at 5 th min post anesthetic treatment, the SV, CO and FS values of the anesthetized fish were significantly decreased in comparison with those of the non-anesthetized fish. However, among the anesthetized fish that were treated with $\mathrm{CB}[7]$ postanesthetic treatment, SV, CO and FS were remarkably improved in comparison with the natural-recovery group post anesthesia. These results suggested that $\mathrm{CB}[7]$ expedited the restoration of cardiac function, thus mitigating the cardiotoxic effects that are commonly induced by general anesthesia.
Meanwhile, there was no obvious difference in the HR, SV, $\mathrm{CO}$ and $\mathrm{FS}$ values between $\mathrm{CB}[7]$ treated group and $\mathrm{E} 3$ medium group. Both of these groups were non-anesthetized fish groups, thus suggesting that this level of $\mathrm{CB}[7](0.5 \mathrm{mM})$ and treatment length exhibited little effect on the cardiac function of zebrafish. Indeed, our recent work show that $0.5 \mathrm{mM} \mathrm{CB}[7]$ exhibited some level of cardiotoxicity in $2 \mathrm{dpf}$ zebrafish larvae after treatment for a much longer period of $2 \mathrm{~d} \cdot .^{22}$ For the purpose of anesthesia reversal, however, only a short exposure period is necessary.

\subsection{Time required to regain equilibrium from anesthesia}

During the staged recovery from anesthesia of fish, stage 3 features the total recovery of equilibrium (ESI Table $2 \dagger$ ). ${ }^{44}$ Equilibrium regaining provides an obvious sign of recovery. Thus we compared the time to regain equilibrium of anesthetized adult zebrafish (adult zebrafish were used for this part of the study for the ease of visual examination of equilibrium), in the absence and in the presence of $0.5 \mathrm{mM} \mathrm{CB}[7]$. The adult zebrafish were anesthetized to stage 4 after $2 \mathrm{mM}$ TM treatment for $10 \mathrm{~min}$. The anesthesia status was confirmed by a characteristic loss of equilibrium, as well the lack of response to spinal pinch. The anesthetized fish were removed from the TM solution and then immediately placed into an E3 medium or a $0.5 \mathrm{mM} \mathrm{CB}[7]$ in E3 medium. The fish were observed to gradually recover and turn to regain their balance, and the time required to regain equilibrium was recorded using a stop watch. As shown in Fig. 7, $\mathrm{CB}[7]$ dramatically reduced the time for anesthetized fish to return to equilibrium to half, from 3 min by natural recovery to $1.5 \mathrm{~min}$ in the presence of $\mathrm{CB}[7]$. In the
(A)

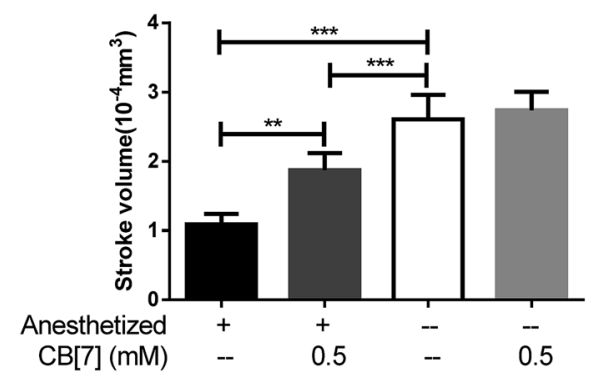

(C)

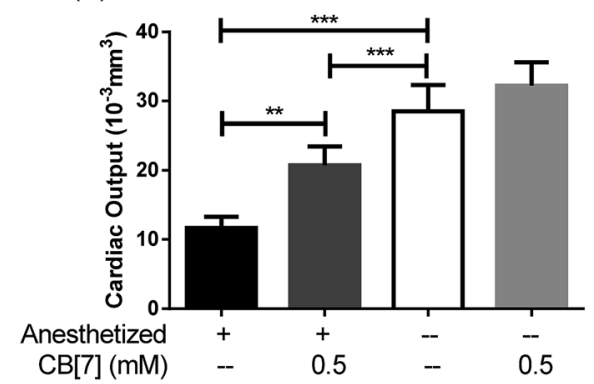

(B)

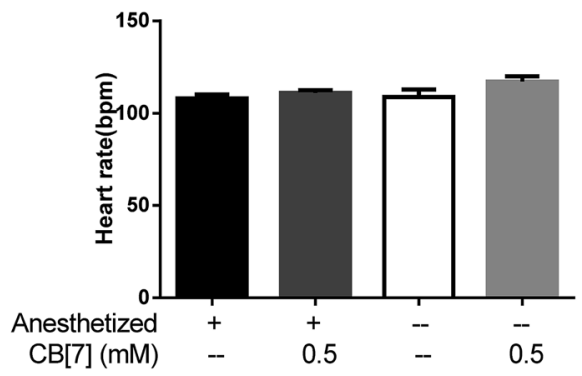

(D)

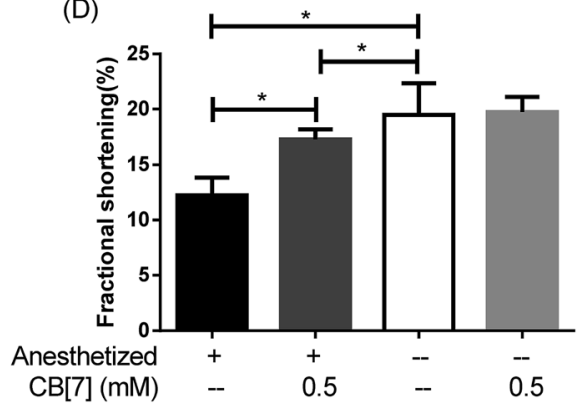

Fig. 6 Cardiac functions of $\mathrm{Tg}(\mathrm{cm} / \mathrm{c2}$ :GFP) zebrafish larvae at 5 min during recovery post anesthesia, in the absence and in the presence of $0.5 \mathrm{mM}$ CB[7]. The stroke volume (A), heart rate (B), cardiac output (C) and \% FS (D) exhibited by the zebrafish were measured after 5 min of recovery. Data are presented as mean \pm S.E.M. $(n=10$ in each group). $* P<0.05, * * P<0.01, * * * P<0.001$. 

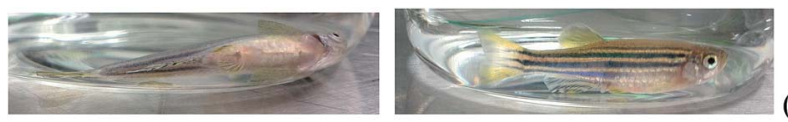

(A)

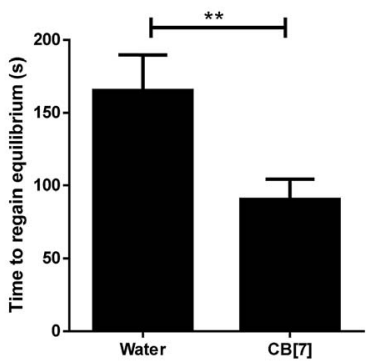

(B)

Fig. 7 Time for adult zebrafish to regain equilibrium after anesthesia. Representative images (side-view) of fish of losing and regaining equilibrium (A); plots of the time recorded (in seconds) for anesthetized fish to regain equilibrium (B). Data are expressed as mean \pm S.E.M. of the results ( $n=12$ in each group). ${ }^{* *} P<0.01$.

control group of fish that were not subjected to anesthesia, exposure to $0.5 \mathrm{mM} \mathrm{CB}[7]$ did not affect the ability of the zebrafish to maintain equilibrium.

\section{Conclusion}

We have studied the in vitro host-guest complexation between $\mathrm{CB}[7]$ and the most commonly used general anesthetic for fish, TM. For the first time we demonstrated the in vivo reversal effect of $\mathrm{CB}[7]$ to general anesthesia with zebrafish models by showing accelerated recovery of locomotion behaviors and cardiac functions of zebrafish larvae, as well as accelerated regaining of equilibrium by adult zebrafish. We speculate that $\mathrm{CB}[7]$ likely acts as a competitive synthetic receptor, encapsulating TM within its lipophilic cavity and thus promoting its dissociation from the Na channels through a concentration gradient that favors the diffusion of TM away from the junction with ion channel into the plasma, thereby relieving its neuro-blocking effect, in a similar manner as exhibited by Sugammadex. ${ }^{7}$ The findings will not only expand the potential applications of $\mathrm{CB}[7]$ in the biomedical field but could also lead to a new approach that may allow patients to regain lucidity much faster than can be achieved through natural recovery from general anesthesia. These supramolecular hosts may also be used to reverse potentially life-threatening toxic effects encountered by some patients in response to general anesthesia.

\section{Experimental}

\subsection{Materials and instrumentation}

Tricaine mesylate (MS-222) and cucurbit[7]uril were both purchased from Sigma-Aldrich Company and used as received. The E3 medium was composed of $5 \mathrm{mM} \mathrm{NaCl}, 0.17 \mathrm{mM} \mathrm{KCl}$, $0.33 \mathrm{mM} \mathrm{CaCl}_{2}$, and $0.33 \mathrm{mM} \mathrm{MgSO}_{4}(\mathrm{pH} 7.2-7.3)$.

The UV-visible spectra were all acquired with a SpectraMax M5 UV-visible spectrometer using quartz cells with a $1.00 \mathrm{~cm}$ path length. The $1 \mathrm{D}{ }^{1} \mathrm{H}$ NMR spectra were recorded using a
Bruker Ultra Shield $400 \mathrm{MHz}$ NMR spectrometer. Molecular modeling was performed with MM2 energy-minimization using the ChemBio3D software package.

Locomotion behaviors of the zebrafish were tracked using the ZebraLab system (Viewpoint Life Sciences, France). The heart morphology, heart rates (HR) and quantitative assessment of other cardiac functions were obtained from video segments that were recorded of individual fish using an Olympus CelîR imaging system consisting of an IX71 microscope at room temperature.

\subsection{Zebrafish maintenance}

Zebrafish (Danio rerio) were kept at the dedicated aquarium area at the Institute of Chinese Medical Sciences, University of Macau, and the procedures for zebrafish culture, breeding, embryo collection, embryonic and larval culture, fluorescent observation were performed according to the standard procedures. ${ }^{45}$ Briefly, adult fish were raised in an aquaculture system with $12 \mathrm{~h}$ light/12 $\mathrm{h}$ dark cycles, and fed twice a day with newly hatched brine shrimp. Mature male and female zebrafish (at a ratio of 1 male to 2 females) were transferred into a breeding tank and separated by a mesh screen the night before breeding. All fertilized embryos were examined under a microscope and those that developed normally were selected for subsequent experiments. Collection of the embryos, and further treatments (with TM and/or $\mathrm{CB}[7]$ ) were all performed in E3 medium. Zebrafish and embryos were maintained at $27 \pm 1{ }^{\circ} \mathrm{C}$. Wild type zebrafish (adults and larvae) of $\mathrm{AB}$ background were used for the behavioral assays, while $\operatorname{Tg}($ cmlc2:GFP) zebrafish with GFP (green fluorescent protein) specifically expressed in the myocardial cells were used for cardiac function assays. ${ }^{46}$ The study protocol was approved by the Animal Ethics Committee of ICMS at the University of Macau. All experiments were conducted according to the ethical guidelines of the ICMS, University of Macau.

\subsection{Locomotion analysis of zebrafish larvae post anesthesia}

Locomotion behaviors of zebrafish larvae post-anesthesia by TM were monitored using the ZebraLab system (Viewpoint Life Sciences, France). From 1-3 dpf (days post-fertilization), zebrafish larvae showed very little spontaneous swimming, but by $5 \mathrm{dpf}$, they spontaneously swam longer distances and independently searched for food. ${ }^{47}$ Thus we chose to use $6 \mathrm{dpf}$ fish for behavior analysis post anesthesia. Zebrafish larvae (6 dpf) were randomly selected and kept individually in each well of a 96-well plate. The E3 medium was removed and $100 \mu \mathrm{L}$ of $1 \mathrm{mM}$ TM (in E3 medium) was added to each well. After $3 \mathrm{~min}$ of incubation, the TM solution was carefully discarded and $100 \mu \mathrm{L}$ of E3 medium or $0.5 \mathrm{mM} \mathrm{CB}[7]$ in E3 medium was added. In addition, non-anesthetized fish (without TM treatment) were used as the control group. Fish locomotion was monitored immediately after the medium addition. Locomotion activity was measured in the tracking mode and the integration time was 60 or 600 seconds. The movement thresholds were set to: inactive if the velocity was $<3 \mathrm{~mm} \mathrm{~s}^{-1}$, low activity if the velocity was $3-6 \mathrm{~mm} \mathrm{~s}^{-1}$, and active if the velocity was $>6 \mathrm{~mm} \mathrm{~s}^{-1}$. 
4.4. Time to regain equilibrium among adult zebrafish post anesthesia

One of the clear indications of partial recovery from anesthesia is that a fish can regain equilibrium in water. ${ }^{37}$ Adult wild type zebrafish were used for this purpose due to the ease of observation of their balancing. The zebrafish were anesthetized with $50 \mathrm{~mL}$ of $2 \mathrm{mM}$ TM in E3 medium for $10 \mathrm{~min}$ to cause them to completely lose their equilibrium. The anesthetized fish were then placed into $50 \mathrm{~mL}$ of $\mathrm{E} 3$ medium or $0.5 \mathrm{mM} \mathrm{CB}$ [7] for recovery, and the time required for this fish to regain their equilibrium (as demonstrated by their ability to remain upright) was recorded.

\subsection{Cardiac function of zebrafish embryos post anesthesia}

$\operatorname{Tg}($ cmlc2:GFP) zebrafish embryos were used as a cardiac function assay due to the myocardial fluorescence that can facilitate cardiac observations. All embryos were cultivated in E3 medium containing $0.003 \mathrm{wt} \%$ of 1-phenyl-2-thiourea (PTU) to block pigmentation since $1 \mathrm{dpf}$. $4 \mathrm{dpf}$ zebrafish embryos were treated with $1 \mathrm{~mL}$ solutions of $1 \mathrm{mM}$ TM in E3 medium. After the zebrafish had been anesthetized for $3 \mathrm{~min}$, the TM solution was removed carefully and replaced with $0.5 \mathrm{mM} \mathrm{CB}[7]$ in $\mathrm{E} 3$ medium or E3 medium alone. After 5 or 20 min of incubation, the zebrafish were immersed into $1 \mathrm{wt} \%$ low-melting point agarose matrix (Gibco) to fix them in a dorsal orientation and restrict their movement. The heart morphology, heart rates (HR) and quantitative assessment of other cardiac functions were obtained from $15 \mathrm{~s}$ video segments recorded of individual fish using an Olympus Cell̂R imaging system equipped with a IX71 microscope at room temperature. Ventricular functions were evaluated according to various parameters, which were measured as described previously. ${ }^{48}$ Briefly, images from the video were used to measure the longitudinal axis length (a) and lateral axis length (b) between the myocardial borders of ventricles at end-diastole and end-systole, respectively. In order to measure the HR, the number of heartbeats in a $15 \mathrm{~s}$ interval was counted. The ventricular volume at end-diastole (EDV) and end-systole (ESV) in the larvae was calculated from the heart dimensions using the formula for a prolate spheroid: $V=$ $4 / 3 \pi a b^{2}$ (ESI Fig. S3†). The stroke volume (SV), cardiac output (CO) and percent fractional shortening (\% FS) were calculated as follows: $\mathrm{SV}=(\mathrm{EDV}-\mathrm{ESV}), \mathrm{CO}=\mathrm{SV} \times \mathrm{HR}, \% \mathrm{FS}=($ diastolic diameter - systolic diameter $) /($ systolic diameter $) \times 100 \%$.

\subsection{Statistical analysis}

Diagrams and statistical analysis using a one-way ANOVA test was performed using GraphPad Prism Software (GraphPad Software, La Jolla CA, USA). The statistical significance of differences between all groups was calculated using the Kruskal-Wallis test. Meanwhile, the Mann-Whitney test was used for comparison between two treatments. $P$-Values of less than 0.05 were considered statistically significant.

\section{Acknowledgements}

We wish to thank the University of Macau UM-SRG (R. W., Project No. SRG2014-00025) for financial support of this project.

\section{Notes and references}

1 E. N. Brown, R. Lydic and N. D. Schiff, N. Engl. J. Med., 2010, 363, 2638-2650.

2 L. D. Gray and C. Morris, Anaesthesia, 2013, 68, 14-29.

3 K. Solt, J. F. Cotten, A. Cimenser, K. F. K. Wong, J. J. Chemali and E. N. Brown, Anesthesiology, 2011, 115, 791-803.

4 M. J. Halsey, Br. J. Anaesth., 1987, 59, 1-2.

5 J. A. Anderson, Anesthesia Program, 1988, 35, 43-47.

6 W. A. Morton and G. G. Stockton, J. Clin. Psychiatry, 2000, 2, 159-164.

7 A. Bom, M. Bradley, K. Cameron, J. K. Clark, J. van Egmond, H. Feilden, E. J. MacLean, A. W. Muir, R. Palin, D. C. Rees and M. Q. Zhang, Angew. Chem., Int. Ed., 2002, 41, 266-270. 8 D. H. Macartney, Future Med. Chem., 2013, 5, 2075-2089.

9 D. Ma, B. Zhang, U. Hoffmann, M. G. Sundrup, M. Eikermann and L. Isaacs, Angew. Chem., Int. Ed., 2012, 51, 11358-11362.

10 U. Hoffmann, M. Grosse-Sundrup, K. Eikermann-Haerter, S. Zaremba, C. Ayata, B. Zhang, D. Ma, L. Isaacs and M. Eikermann, Anesthesiology, 2013, 119, 317-325.

11 K. I. Assaf and W. M. Nau, Chem. Soc. Rev., 2015, 44, 394-418. 12 F. J. McInnes, N. G. Anthony, A. R. Kennedy and N. J. Wheate, Org. Biomol. Chem., 2010, 8, 765-773.

13 N. J. Wheate, V. Vora, N. G. Anthony and F. J. McInnes, J. Inclusion Phenom. Macrocyclic Chem., 2010, 68, 359-367.

14 N. J. Wheate, D. P. Buck, A. I. Day and J. G. Collins, Dalton Trans., 2006, 451-458.

15 A. R. Kennedy, A. J. Florence, F. J. McInnes and N. J. Wheate, Dalton Trans., 2009, 7695-7700.

16 N. J. Wheate, J. Inorg. Biochem., 2008, 102, 2060-2066.

17 I. W. Wyman and D. H. Macartney, Org. Biomol. Chem., 2010, 8, 247-252.

18 R. Wang and D. H. Macartney, Org. Biomol. Chem., 2008, 6, 1955-1960.

19 R. Wang, D. Bardelang, M. Waite, K. A. Udachin, D. M. Leek, K. Yu, C. I. Ratcliffe and J. A. Ripmeester, Org. Biomol. Chem., 2009, 7, 2435-2439.

20 M. A. Gamal-Eldin and D. H. Macartney, Can. J. Chem., 2014, 92, 243-249.

21 Y. Gibert, M. C. Trengove and A. C. Ward, Curr. Med. Chem., 2013, 20, 2458-2466.

22 H. Chen, J. Y. W. Chan, X. Yang, I. W. Wyman, D. Bardelang, D. H. Macartney, S. M. Y. Lee and R. Wang, RSC Adv., 2015, 5, 30067-30074.

23 A. J. Hill, H. Teraoka, W. Heideman and R. E. Peterson, Toxicol. Sci., 2005, 86, 6-19.

24 R. T. Peterson, R. Nass, W. A. Boyd, J. H. Freedman, K. Dong and T. Narahashi, Neurotoxicology, 2008, 29, 546-555.

25 P. I. Bang, P. C. Yelick, J. J. Malicki and W. F. Sewell, J. Neurosci. Methods, 2002, 118, 177-187.

26 C. Parng, C. Ton, Y. X. Lin, N. M. Roy and P. McGrath, Neurotoxicol. Teratol., 2006, 28, 509-516.

27 N. S. Sipes, S. Padilla and T. B. Knudsen, Birth Defects Res., Part C, 2011, 93, 256-267. 
28 D. T. Frazier and T. Narahashi, Eur. J. Pharmacol., 1975, 33, 313-317.

29 M. Matthews and Z. M. Varga, ILAR J., 2012, 53, 192-204.

30 J. F. T. Butterworth and G. R. Strichartz, Anesthesiology, 1990, 72, 711-734.

31 J. F. Burka, K. L. Hammell, T. E. Horsberg, G. R. Johnson, D. J. Rainnie and D. J. Speare, J. Vet. Pharmacol. Ther., 1997, 20, 333-349.

32 S. Attili and S. M. Hughes, PLoS One, 2014, 9, e103751.

33 H. A. D. P. Tytler, Vivisection, anaesthetics and minor surgery, Aquarium systems, ed. A. D. Hawkins, Academic Press, New York, NY, USA, 1981.

34 I. W. Wyman and D. H. Macartney, Org. Biomol. Chem., 2010, 8, 1796-1801.

35 P. Job, Ann. Chim. Appl., 1928, 9, 113-203.

36 E. Masson, X. Ling, R. Joseph, L. Kyeremeh-Mensah and X. Lu, RSC Adv., 2012, 2, 1213-1247.

37 J. Nordgreen, F. M. Tahamtani, A. M. Janczak and T. E. Horsberg, PLoS One, 2014, 9, e92116.

38 V. M. T. V. Anna Mercy and S. Sajan, Journal of Threatened Taxa, 2013, 5, 4414-4419.

39 B. M. Heath, Y. Cui, S. Worton, B. Lawton, G. Ward, E. Ballini, C. P. Doe, C. Ellis, B. A. Patel and
N. C. McMahon, J. Pharmacol. Toxicol. Methods, 2011, 63, 258-268.

40 R. Letienne, B. Vie and B. le Grand, Eur. J. Pharmacol., 2006, 530, 243-249.

41 M. F. Nilsson, H. Ritchie and W. S. Webster, Birth Defects Res., Part B, 2013, 98, 416-427.

42 M. P. Craig, S. D. Gilday and J. R. Hove, Lab. Anim., 2006, 35, 41-47.

43 M. A. Denvir, C. S. Tucker and J. J. Mullins, BMC Biotechnol., 2008, 8, 21.

44 Y. Hikasa, K. Takase, T. Ogasawara and S. Ogasawara, Jpn. J. Vet. Sci., 1986, 48, 341-351.

45 M. Westerfield, The Zebrafish Book: A Guide for the Laboratory Use of Zebrafish (Danio rerio), University of Oregon Press, 2000.

46 C. J. Huang, C. T. Tu, C. D. Hsiao, F. J. Hsieh and H. J. Tsai, Dev. Dyn., 2003, 228, 30-40.

47 W. Cui, Z. Zhang, W. Li, S. Hu, S. Mak, H. Zhang, R. Han, S. Yuan, S. Li, F. Sa, D. Xu, Z. Lin, Z. Zuo, J. Rong, E. D. Ma, T. C. Choi, S. M. Lee and Y. Han, Br. J. Pharmacol., 2013, 168, 1201-1214.

48 Z. Chen, W. Huang, T. Dahme, W. Rottbauer, M. J. Ackerman and X. Xu, Cardiovasc. Res., 2008, 79, 97-108. 